\title{
Evaluation of the Risk of Spreading Fire Blight in Apple Orchards with a Mechanical String Blossom Thinner
}

\author{
Henry K. Ngugi ${ }^{1}$ \\ Department of Plant Pathology, Penn State University Fruit Research and \\ Extension Center, 290 University Drive, P.O. Box 330, Biglerville, PA 17307
}

James R. Schupp

Department of Horticulture, Penn State University Fruit Research and Extension Center, Biglerville, PA 17307

Additional index words. crop load management, risk assessment, blossom thinning, Malus domestica

\begin{abstract}
The risk of spreading fire blight in apples after mechanical thinning with a rotating string blossom thinner was evaluated in field and potted-tree experiments. In the field experiment, using the mechanical thinner on noninoculated trees immediately after operating the equipment on inoculated trees significantly $(P<0.01)$ increased fire blight incidence resulting in $90 \pm \mathbf{2 0 . 0 1}$ (mean $\pm \mathrm{SE}$ ) infected shoots compared with $23.5 \pm \mathbf{8 . 9 7}$ diseased shoots in similar trees that were not thinned mechanically. A similar result was obtained in greenhouse experiments whereby healthy apple plants positioned adjacent to diseased plants before the group was subjected to the mechanical thinner developed more than twice the number of infected shoots as that on similar plants that were not thinned. These results indicate that under conditions conducive to infection, the mechanical blossom thinner significantly increases the risk of spreading Erwinia amylovora. The use of the thinner should therefore be limited to orchards with no history of disease in the last 3 years and on days when predicted weather is not suitable for tree infection by E. amylovora; otherwise, a severe fire blight epidemic could develop in the orchard.
\end{abstract}

Interest in economical nonchemical thinner alternatives for use in the tree fruit industry is increasing as the supply of farm workers continues to decline and labor costs increase. In 2007, Penn State researchers tested a mechanical string thinner for blossom thinning of fruit trees (Schupp et al., 2008). Several extensive trials were conducted in commercial peach orchards, but as a result of concerns about fire blight, only one preliminary trial was conducted on apple at the Penn State Fruit Research and Extension Center. Used at pink, the thinner effectively reduced fruit set, decreased time required for follow-up hand thinning, and increased fruit size of 'GoldRush'/M.26 apple trees. Although no fire blight was observed in the 2007 trial, we observed the rotating nylon strings of the mechanical blossom thinner not only removed apple flowers, but also shredded primary spur leaves, leaving many fresh wounds. Apple flowers develop in a mixed bud containing both vegetative and reproductive tissues. Furthermore, because apple flowers have a long pedicel, considerable velocity is required

Received for publication 8 Dec. 2008. Accepted for publication 18 Feb. 2009.

This research was funded by the State Horticultural Association of Pennsylvania (SHAP).

We thank Emily Pfeufer, Katie Hess-Reichard, and Margaret E. Reid for technical support.

${ }^{1}$ To whom reprint requests should be addressed; e-mailhkn3@psu.edu. for the cord to slice away the flower tissue. The resulting tissue damage combined with the repeated contact of the mechanical thinner with the tree canopy raises the question as to whether the thinner can transmit the fire blightcausing bacterium Erwinia amylovora (Burrill) Winslow et al., thereby increasing disease risk.

The manufacturer of the rotating string thinner reported that in Germany, the incidence and severity of fire blight was no worse or better in mechanically thinned orchards than that observed in adjacent blocks where the thinner was not used (Adolf Betz, FruitTec, personal communication). However, the environmental conditions in the mid-Atlantic region of the United States at bloom are likely to be more conducive to fire blight development than in western Europe, which has a cooler maritime climate. Bertschinger et al. (1998) noted that string thinning at bud swell severely damaged shoots and spurs of 'Jonica'. They reported that the best results were obtained between the tight cluster and pink stages, because timing near full bloom resulted in deformed fruit and increased the possibility of spreading disease, especially fire blight (E. amylovora), and concluded that the severity of undesirable damage can be lessened by using the string thinner between tight cluster and pink, before spur leaf expansion is complete.

The objective of this research was to evaluate the risk of spreading $E$. amylovora by mechanical blossom thinning from blossom infections on bearing trees in the field and from shoot blight infections on young trees grown in the greenhouse.

\section{Materials and Methods}

Thinning applications were made with a rotating string thinner (Darwin 300; FruitTec, Deggenhausertal, Germany) designed to remove apple blossoms in organic orchards (Bertschinger et al., 1998). The string thinner consisted of a tractor-mounted frame with a $3.0 \mathrm{~m}$ tall vertical spindle in the center of the frame. Attached to the spindle were 36 steel plates securing a total of 648 plastic cords each measuring $50 \mathrm{~cm}$ in length. Speed of the clockwise rotating spindle was adjusted with a hydraulic motor. The string thinner spindle was operated at $245 \mathrm{rpm}$, and the mechanical thinner was driven through orchard rows at $2.4 \mathrm{~km} \cdot \mathrm{h}^{-1}$.

Inoculum for both field and potted plant experiments was prepared from cultures of $E$. amylovora strain Ea273 obtained from Dr. T.W. McNellis, Department of Plant Pathology, Penn State University, University Park, PA. Bacterial cultures were stored in $20 \%$ glycerol at $-80{ }^{\circ} \mathrm{C}$ and fresh inoculum prepared as needed. Inoculum for each experiment was prepared from fresh, 48-h-old cultures grown on modified Lennox medium (Jensen et al., 2003). Bacterial suspensions were made by flooding plates with $0.05 \mathrm{M}$ sterile potassium phosphate buffer $(\mathrm{pH} 7.4)$ and agitating slowly for $5 \mathrm{~min}$. Bacterial suspensions were then diluted to the appropriate optical density at $600 \mathrm{~nm}\left(\mathrm{OD}_{600 \mathrm{~nm}}\right)$. Optical density was measured with a Hach DR/4000U spectrophotometer (Hach Company, Loveland, CO).

Field experiment. The field experiment was carried out on mature 'York Imperial'/ MM.106 apple trees growing at the Penn State Fruit Research and Extension Center. The trees are at least 20 years old and trained to a central leader system. Data on fire blight incidence were obtained from noninoculated trees. Experimental treatments depended on the position of the evaluated trees relative to that of inoculated trees and on whether the test trees were thinned or not thinned with the mechanical thinner immediately after the equipment had been used on inoculated trees. The specific treatments consisted of: 1) control trees that were neither inoculated nor thinned (control); 2) noninoculated trees that were adjacent to inoculated trees and thinned with the mechanical blossom thinner immediately after the equipment had been used on the inoculated trees (inoculation + thinner); 3 ) noninoculated trees that were adjacent to inoculated trees but were not thinned with the mechanical blossom thinner (inoculation + no thinner); and 4) noninoculated trees that were thinned with the mechanical blossom thinner (thinner + no inoculation). The treatments were arranged in a randomized complete block design and four replicate trees were used for each treatment. Trees in Treatments 2 and 3 were spray-inoculated with a 2 $\times 10^{8}$ colony-forming unit (CFU) suspension of E. amylovora strain Ea273 at 75\% bloom. 
Thinning with the mechanical blossom thinner was carried out on 6 May, $7 \mathrm{~d}$ after inoculation with early fire blight symptoms having developed on the inoculated trees. To prevent crosscontamination, treatments involving inoculated trees were executed after those without inoculation. Immediately after thinning, the foliage of all trees was thoroughly moistened by applying water with a boom sprayer to simulate a rainfall event. Daily maximum temperatures for the 2 weeks after inoculation exceeded $18^{\circ} \mathrm{C}$. Treatments were monitored for shoot blight symptoms and the number of infected shoots per tree was counted 2 weeks after mechanical thinning.

Potted tree experiment. To evaluate the threat of spreading shoot blight on nonflowering trees, experiments were carried out with 2-year-old 'Royal Gala' and 'Golden Delicious'/‘Budagovsky 9' trees maintained in $29-\mathrm{cm}$ diameter plastic pots in the greenhouse. The trees were 1.75 to $2.0 \mathrm{~m}$ tall with a canopy diameter $\approx 75 \mathrm{~cm}$ wide at the base. Experimental treatments consisted of: 1) untreated control trees that were neither inoculated nor thinned with the mechanical blossom thinner (control); 2) healthy plants that were placed adjacent to diseased plants before the group being thinned with the mechanical blossom thinner (inoculation + thinner); 3) healthy plants that were placed adjacent to diseased plants but which were not thinned with the mechanical blossom thinner (inoculation + no thinner); and 4) healthy plants that were placed adjacent to healthy plants before being thinned with the mechanical blossom thinner (thinner + no inoculation). The experimental design was a two-factor arrangement with thinning treatment and apple cultivar as factors. Four replicate plants of each cultivar were used for each thinning treatment.

Fire blight on diseased plants was initiated by inoculating four young shoots of each plant with a $1 \times 10^{8} \mathrm{CFU}$ suspension of $E$. amylovora strain Ea273 and maintaining the plants at high humidity for $10 \mathrm{~d}$ in the greenhouse before the thinning experiments. Inoculation was done by excising from the distal end $2 \mathrm{~cm}$ of tissue on the two youngest leaves of apical shoots with a pair of scissors dipped in a beaker containing the bacterial suspension. To carry out mechanical thinning, plants were removed from the greenhouse, placed on flat ground, and secured in place with a steel wire positioned $\approx 0.5 \mathrm{~m}$ horizontally above the pot soil level. The tension in the wire was maintained by hooking it to two farm tractors and pulling in opposite directions. Plants were positioned such that individual treatments were sufficiently separated by at least $5 \mathrm{~m}$ of empty space along the wire and treatments involving inoculated trees were executed last. Immediately before and after thinning the plants with the mechanical blossom thinner, the foliage of plants in all experimental treatments was kept moist by sprinkling them with water for $\approx 5$ min with a garden hose. After application of the thinning treatments, plants were returned to the greenhouse and the foliage was moistened at least once each day. Plants were assessed for fire blight incidence (number of shoots with symptoms) and severity $14 \mathrm{~d}$ after application of the thinning treatments. Fire blight severity was rated on a 1 to 7 scale in which: $1=$ no symptoms; 2 = initial systemic infection with water soaking but no necrosis; $3=$ systemic infection with necrosis restricted to the midrib; $4=$ necrosis on at least one leaf; $5=$ necrosis on at least two leaves with initial shoot wilt symptoms; $6=$ systemic infection and wilt of the shoot with at least three necrotic leaves and with or without bacterial ooze; and $7=$ severe symptoms with at least five apical leaves infected and the shoot showing characteristic wilt symptoms.

Data on incidence of shoot infection from the field experiment were subjected to a square root transformation before analysis. Both data sets were analyzed with the Proc GLM procedure of SAS release 9.1 (SAS Institute Inc., Cary, NC). Means were separated with Fisher's protected least significant difference test $(\alpha=0.01)$

\section{Results}

Field experiment. Thinning apple trees with the mechanical blossom thinner significantly $(P<0.01)$ increased the incidence of fire blight on shoots. Trees thinned with the mechanical blossom thinner immediately after the equipment was used on inoculated trees developed greater than 90 infected shoots per tree. This was more than three times the number of diseased shoots $(23.5 \pm$ 8.97) that developed on trees in similar proximity to inoculated trees but which were not subjected to the mechanical thinner treatment (Fig. 1). The incidence of infected shoots for trees that were not inoculated but subjected to thinning with the mechanical blossom thinner was $9.8 \pm 2.75$. Although numerically larger, this value was not signif- icantly greater $(P=0.152)$ than that obtained in the untreated control trees $(4.8 \pm 2.63$; Fig. 1). In general, the mechanically thinned trees had visibly wounded shoots and spurs.

Potted tree experiment. Statistical analysis revealed that there were no differences between apple cultivars in the incidence of shoots with fire blight or in disease severity after the mechanical blossom thinner treatments. The means of the two cultivars were therefore pooled for presentation. Fire blight symptoms developed on an average of five shoots on healthy trees that were placed adjacent to inoculated trees before the group being subjected to the mechanical blossom thinner treatment (Table 1). By contrast, only one shoot $($ mean $=0.13 \pm 1.38$; Table 1$)$ on 'Golden Delicious' developed fire blight symptoms when healthy plants were placed adjacent to inoculated plants but not subjected to simulated thinning with the mechanical thinner. Three shoots developed fire blight symptoms when healthy plants that were placed adjacent to untreated plants were subjected to simulated thinning with a mechanical blossom thinner. Although not statistically greater, this number was more than twice that of similar trees that were not subjected to the mechanical thinner, suggesting that wounds created by the thinner exposed the plants to infection even when plants were not in direct contact with diseased plants.

Like with incidence, fire blight severity was greatest on shoots of plants placed adjacent to inoculated trees before the group being subjected to the simulated thinning with the mechanical blossom thinner (Table $1)$. Infected shoots of plants in this category developed characteristic fire blight symptoms with systemic infection in some shoots affecting greater than five leaves (disease severity score 7) culminating in the typical shepherd's crook (Fig. 2A) by the time of assessment. Close inspection of diseased

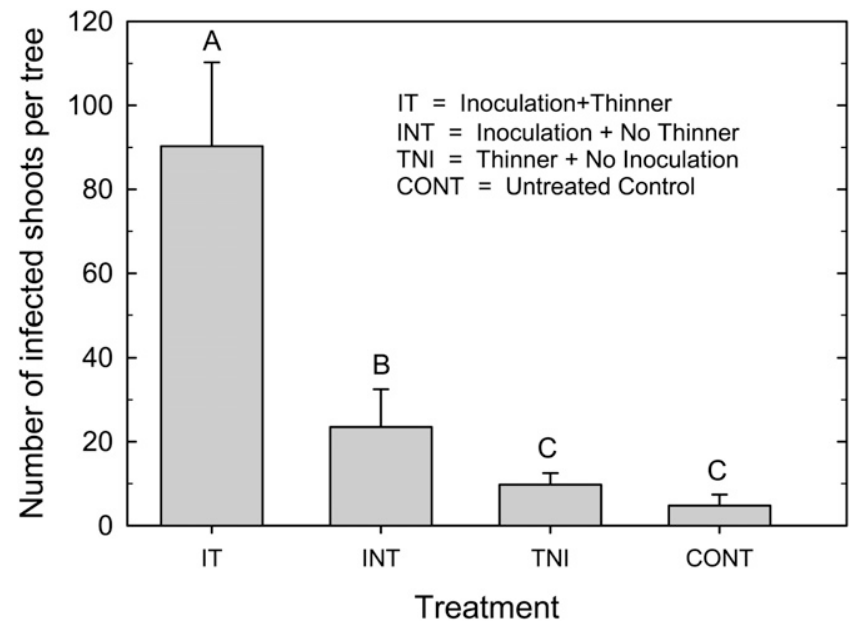

Fig. 1. Number of shoots with fire blight symptoms 2 weeks after trees were subjected to different inoculation and thinning treatments with a mechanical blossom thinner in a field experiment evaluating the risk of spreading Erwinia amylovora with the mechanical thinner. Values are means and SEs of four replicate trees; means with the same letter are not significantly different based on Fisher's protected least significant difference $(\alpha=0.01)$. 
Table 1. Mean number of shoots with fire blight symptoms and disease severity on 2-year-old potted 'Gala' and 'Golden Delicious' trees subjected to different mechanical thinning treatments with a rotating string blossom thinner.

\begin{tabular}{lcc}
\hline Treatment $^{\mathrm{z}}$ & Number of strikes $^{\mathrm{y}}$ & Fire blight severity $^{\mathrm{yx}}$ \\
\hline Inoculation + thinner & $5.00 \mathrm{~A}$ & $5.25 \mathrm{~A}$ \\
Inoculation + no thinner & $0.13 \mathrm{~B}$ & $1.38 \mathrm{~B}$ \\
Thinner + no inoculation & $0.38 \mathrm{~B}$ & $1.25 \mathrm{~B}$ \\
Untreated control & $0.00 \mathrm{~B}$ & $1.00 \mathrm{~B}$ \\
\hline
\end{tabular}

${ }^{\mathrm{z}}$ Healthy plants were positioned adjacent to inoculated or noninoculated trees before the group was subjected to the mechanical blossom thinner treatment.

${ }^{y}$ Values are means of eight replicate trees. Means in the same column followed by the same letter are not significantly different based on Fisher's protected least significant difference test $(\alpha=0.01)$.

${ }^{\mathrm{x}}$ Severity rated on a 1 to 7 scale in which $1=$ no disease symptoms and $7=$ severe symptoms with systemic shoot wilt symptoms and at least five fully expanded leaves dead.
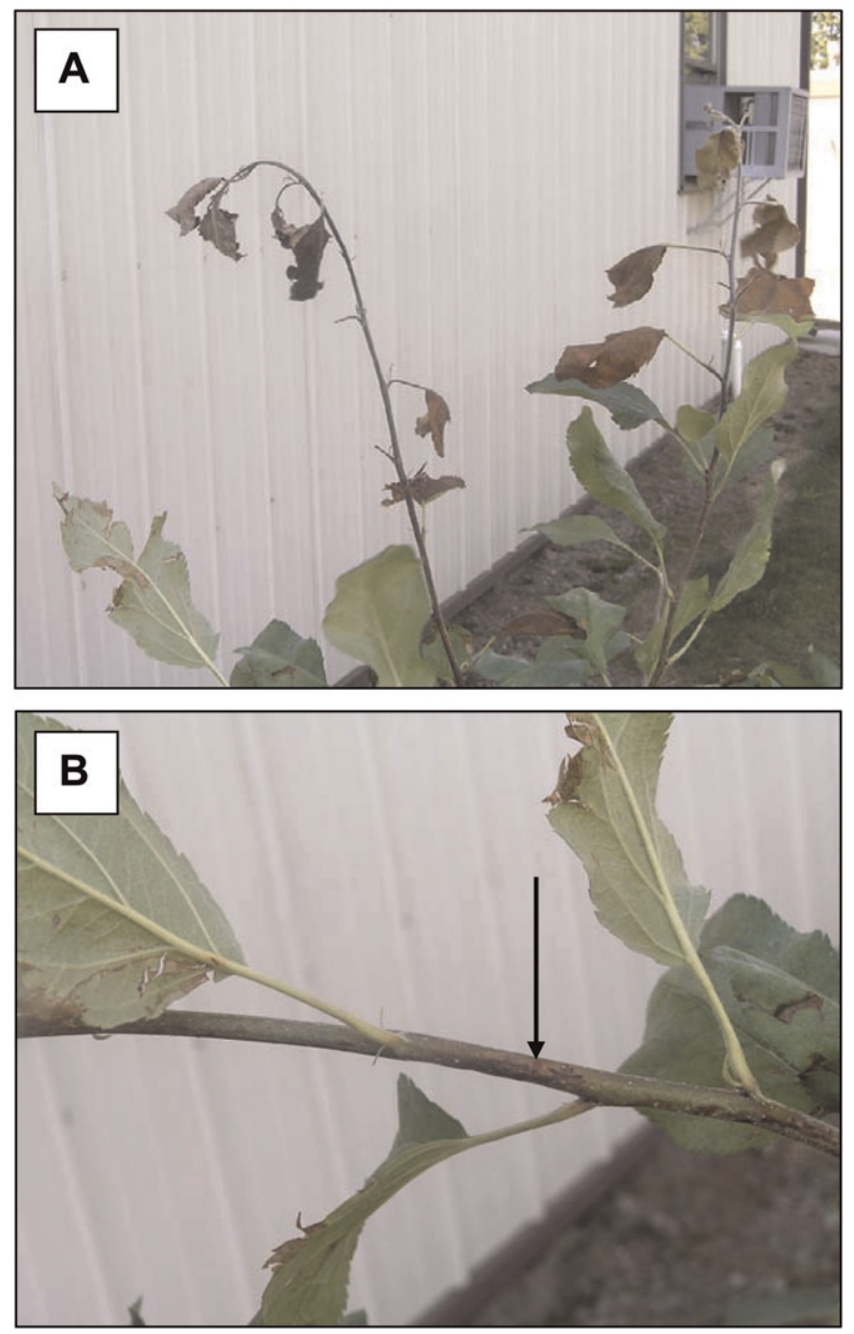

Fig. 2. A shoot with shredded leaves and characteristic shepherd's crook wilt symptoms of fire blight after simulated mechanical thinning with the mechanical blossom thinner (A) and a closeup of a wound caused by the mechanical thinner showing bacterial ooze symptoms (arrow) typical of infections by Erwinia amylovora $(\mathbf{B})$.

shoots in this treatment revealed that infections appeared to originate from wounds caused by the spinning twines of the mechanical thinner (Fig. 2B).

\section{Discussion}

In this study, we assessed the potential of a mechanical string blossom thinner to spread E. amylovora on apples under conditions suitable for disease development. Our results indicate that under the test conditions, sub- et al., 2006; Norelli et al., 2003), the incidence of shoot strikes was higher in plants that received the thinning treatments indicating that the mechanical thinner did indeed increase the likelihood of infection.

Several factors must coincide and be optimal for a fire blight epidemic to occur. These include: presence of inoculum; susceptible host tissue (open receptive flowers for blossom strikes and wounded tissue for shoot strikes); weather conditions suitable for infection (moisture and temperatures above $15^{\circ} \mathrm{C}$ ); and although less important for shoot strikes, presence of a suitable vector (Billing 2007; Ngugi, 2008; Thomson, 2000). Our tests provided optimal conditions for infection to increase the likelihood of capturing treatment effects. It is unlikely that such conditions would always be present during normal apple-thinning operations. For example, inoculum pressure in commercial orchards would likely be lower than that provided in our experiments, and Bertschinger et al. (1998) recommended that a mechanical blossom thinner is best used at the pink bud stage when temperatures are likely to be limiting for fire blight development (Billing, 1974, 2007). Nevertheless, documenting increased risk associated with the mechanical thinner is essential for supporting precautionary advice to fruit growers. The mechanical thinner caused substantial tissue wounding and such wounds may remain open to infection albeit at a less frequent rate when the inoculum pressure is low. Once established, fire blight epidemics can develop very rapidly at the onset of optimal conditions, especially on young trees.

In deciding whether to use a mechanical blossom thinner, it is important to consider the mechanism by which the thinner exacerbates the fire blight risk. Because plants treated with the mechanical thinner suffer visible wounds on leaf and cambium tissue, it is reasonable to assume that the wounds serve as an infection court for E. amylovora. A mechanical blossom thinner therefore increases the number of potential infection courts akin to damage caused by hail or storm events (Norelli et al., 2003). Based on these observations, there are several recommendations that may mitigate the risk of fire blight epidemics resulting from use of the mechanical thinner. First, weather forecasting models should be used to predict the risk of infection; where possible, the use of the mechanical thinner should be avoided on days when temperature and moisture are predicted to favor wound infection and bacterial multiplication. Second, the mechanical thinner should not be used in orchards or blocks with a history of disease because they are likely to contain residual inoculum. Finally, if the mechanical thinner has been used and conditions subsequently develop that favor fire blight development, a prophylactic application of a bactericide similar to that recommended after a storm event (Ngugi, 2008; Norelli et al., 2003) should be made. 


\section{Literature Cited}

Bertschinger, L., W. Stadler, F.P. Weibel, and R. Schumacher. 1998. New methods for an environmentally safe regulation of flower and fruit set and of alternate bearing of the apple crop. Acta Hort. 466:65-70.

Billing, E. 1974. The effect of temperature on the growth of the fire blight pathogen, Erwinia amylovora. J. Appl. Bacteriol. 37:643-648.

Billing, E. 2007. Challenges in adaptation of plant disease warning systems to new locations: Reappraisal of Billing's integrated system for predicting fire blight in a warm dry environment. Phytopathology 97:1036-1039.
Jensen, P.J., J. Rytter, E.A. Detwiler, J.W. Travis, and T.W. McNellis. 2003. Rootstock effects on gene expression patterns in apple tree scions. Plant Mol. Biol. 53:493-511.

Johnson, K.B. and V.O. Stockwell. 1998. Management of fire blight: A case study in microbial ecology. Annu. Rev. Phytopathol. 36:227-248.

Maccagnani, B., C. Bazzi, E. Biondi, D. Tesoriero, and S. Maini. 2006. Potential of Osmia cornuta as a carrier of antagonistic bacteria in biological control of fire blight: A comparison with Apis mellifera. Acta Hort. 704:379-386.

Ngugi, H.K. 2008. Pennsylvania fire blight watch. The Fruit Times 27:1-2. <http://fruittimes.cas. psu.edu/FT2704.pdf>.
Norelli, J.L., A.L. Jones, and H.S. Aldwinckle. 2003. Fire blight management in the twentyfirst century: Using new technologies that enhance host resistance in apple. Plant Dis. 87:756-765.

Schupp, J.R., T. Auxt Baugher, S.S. Miller, R.M. Harsh, and K.M. Lesser. 2008. Mechanical thinning of peach and apple trees reduces labor input and increases fruit size. HortTechnology 18:660-670.

Thomson, S.V. 2000. Epidemiology of fire blight, p. 9-36. In: Vanneste, J.L. (ed.). Fire blight: The disease and its causative agent, Erwinia amylovora. $\mathrm{CAB}$ International, Wallingford, UK. 